\title{
Usefulness of neutrophil to lymphocyte ratio in patients with chronic obstructive pulmonary disease: a prospective observational study
}

Seung Jun Lee ${ }^{1}$, Hyang Rae Lee ${ }^{1}$, Tae Won Lee ${ }^{1}$, Sunmi Ju ${ }^{1}$, Sujin Lim ${ }^{1}$, Se-Il Go ${ }^{2}$, Jung-Wan You ${ }^{1}$, Yu Ji Cho ${ }^{1}$, Gyeong-Won Lee ${ }^{2}$, Yi Yeong Jeong ${ }^{1}$, Ho Cheol Kim ${ }^{1}$, and Jong Deog Lee ${ }^{1}$

Divisions of ${ }^{1}$ Pulmonology and Allergy and ${ }^{2} \mathrm{Hematology}$ and Oncology, Department of Internal Medicine, Gyeongsang National University Hospital, Gyeongsang National University School of Medicine, Jinju, Korea
Received: March 31, 2015

Revised : May 13, 2015

Accepted: May 22, 2015

\section{Correspondence to}

Jong Deog Lee, M.D.

Division of Pulmonology and

Allergy, Department of Internal Medicine, Gyeongsang National University Hospital, Gyeongsang National University School of

Medicine, 79 Gangnam-ro, Jinju 52727 , Korea

Tel: $+82-55-750-8611$

Fax: $+82-55-758-9122$

E-mail:1jd8611@nate.com
Background/Aims: Neutrophil to lymphocyte ratio (NLR) in peripheral blood is a useful systemic inflammatory response biomarker. However, NLR has not been studied in patients with chronic obstructive pulmonary disease (COPD). This study was aimed to evaluate the usefulness of NLR in patients with COPD.

Methods: NLR was prospectively measured and compared in patients with COPD exacerbation $(n=59)$, patients with stable COPD $(n=61)$, and healthy controls ( $n$ $=28$ ). NLR in patients with COPD exacerbation was repeatedly measured in the convalescent period. The correlation between NLR and clinical parameters was evaluated, and the predictors for respiratory hospitalization were analyzed by multivariate logistic regression.

Results: NLR values were significantly higher in patients with COPD exacerbation compared with stable COPD patients and controls $(12.4 \pm 10.6,2.4 \pm 0.7,1.4$ \pm 0.5 , respectively; $p<0.001$ ). NLR was significantly decreased during the convalescent period in patients with COPD exacerbation ( $4.5 \pm 4.6$ vs. $11.5 \pm 8.8, p<$ $0.001)$. NLR exhibited a significant correlation with the body mass index, degree of airway obstruction, dyspnea, and exercise capacity (BODE) index, the 6-minute walk test, and the modified Medical Research Council scale. NLR $\geq 2.8$ was an independent predictor with a borderline significance for respiratory hospitalization (odds ratio, 2.083; $p=0.079$ ). Body mass index and forced expiratory volume in 1 second were independent predictors for respiratory hospitalization.

Conclusions: NLR is a straightforward and effective biomarker of COPD exacerbation that may serve as a predictor for respiratory hospitalization in patients with COPD.

Keywords: Neutrophil to lymphocyte ratio; Pulmonary disease, chronic obstructive; Usefulness; Respiratory hospitalization

\section{INTRODUCTION}

Chronic obstructive pulmonary disease (COPD) is the fourth leading cause of death worldwide, with death rates reaching 3 million individuals in 2011 [1]. Chron- ic airway inflammation, followed by structural changes and narrowing of the small airways, is a key pathogenic mechanism of COPD [2]. There is a growing interest in pulmonary biomarkers as a mechanism to understand and monitor airway inflammation in patients with 
COPD [3-5]. Systemic inflammatory response is also a major component of COPD, and is closely related to comorbidities of patients with COPD [6-8]. Recently, the neutrophil to lymphocyte ratio (NLR) in peripheral blood has garnered attention as a potential systemic inflammatory marker. NLR has been used as an independent prognostic factor in various solid tumors, including lung cancer, colorectal cancer, pancreatic cancer, breast cancer, ovarian cancer, and gastric cancer [9-14]. Furthermore, cardiovascular outcomes could be independently predicted by NLR in patients with various cardiovascular diseases (CVDs) [15,16]. However, studies on the role of NLR in patients with COPD are limited, despite the fact that COPD is a chronic inflammatory disease.

The aim of this prospective observational study was to evaluate the potential role of NLR in patients with COPD. We evaluated the potential for NLR to serve as a biomarker of COPD exacerbation, as well as whether NLR could predict the need for respiratory hospitalization.

\section{METHODS}

\section{Study patients}

Patients admitted to Gyeongsang National University Hospital, Jinju, South Korea for COPD exacerbation, and outpatients with stable COPD were prospectively enrolled between January 2010 and April 2011. The control group was comprised of gender and smoking history matched healthy subjects who visited a health promotion center to have regular checkups. The Gyeongsang National University Hospital Institutional Review Board approved the current study.

COPD was diagnosed by spirometry if the ratio of post-bronchodilator forced expiratory volume in 1 second to forced vital capacity $\left(\mathrm{FEV}_{1} / \mathrm{FVC}\right)$ was $<0.7$ according to the Global Initiative for Chronic Obstructive Lung Disease guidelines. An exacerbation of COPD was defined as acute deterioration of the patient's respiratory symptoms that were beyond normal day-today variations, and required additional steroids or antibiotics. The convalescence period of COPD exacerbation was determined by a clinician when the patient's condition was stable after discharge at the outpatient department.
Patients with stable COPD were enrolled if they had no history of acute exacerbation during the past 3 months, and were not prescribed long-term oxygen therapy or regular oral corticosteroids.

Exclusion criteria for subject enrollment included: (1) age of less than 40 years; (2) smoking history of less than 10 pack years; (3) severe structural lung disease such as bronchiectasis or lungs destroyed by tuberculosis; (4) bronchial asthma; (5) a concurrent active inflammatory disease other than COPD; or (6) uncontrolled malignant neoplasm.

\section{Study design}

Peripheral venous blood of patients with COPD exacerbation was collected, and NLR during the exacerbation period was measured within 24 hours of admission. NLR values during the convalescent period, when patients' condition was considered clinically stable, were measured on the day of the first outpatient visit after discharge. NLR values were compared between patients with COPD exacerbation, patients with stable COPD, and controls. Changes in NLR between the exacerbation period and the convalescent period in patients with COPD exacerbation were likewise assessed.

The body mass index, degree of airway obstruction, dyspnea, and exercise capacity (BODE) index was calculated if the patient agreed to perform a 6-minute walk test $(6 \mathrm{MWT})$. The $6 \mathrm{MWT}$ was performed by patients with COPD exacerbation during the convalescent period, and by patients with stable COPD. The correlation between NLR and clinical parameters including body mass index (BMI), $\mathrm{FEV}_{1}$, the modified Medical Research Council scale (mMRC), 6MWT, and the BODE index was assessed.

All of the patients were monitored regularly until the last outpatient visit. Hospital readmission of patients with COPD exacerbation, and admission of patients with stable COPD due to COPD exacerbation were recorded. The cut-off value for NLR for predicting hospital readmission in patients with COPD exacerbation, and admission in patients with stable COPD, was determined using a receiver operating characteristic (ROC) curve. NLR in patients with stable COPD, and NLR in the convalescent period of COPD exacerbation patients, were used to determine the cut-off value to predict admission. The predicting factors for respiratory hospital- 
ization were analyzed.

\section{Statistical analysis}

The Pearson chi-square test was used to analyze categorical variables. Comparison of continuous variables between patients with COPD exacerbation, stable COPD, and controls was performed using one-way analysis of variance with the Bonferroni post hoc test. Comparison of continuous variables between two groups was performed using an unpaired $t$ test. A paired $t$ test was used to compare the change in NLR between the exacerbation and convalescent periods. The correlation coefficients and significance of continuous variables were analyzed using Pearson correlation test. ROC curves were constructed, and area under the curve was obtained to determine the optimal cut-off value for NLR for respiratory hospitalization. Independent risk factors for respiratory hospitalization were analyzed using multivariate binary logistic regression. Odds ratios (ORs) and 95\% confidence intervals were estimated. Statistical signif- icance was accepted at $p<0.05$. All statistical analyses were performed using SPSS version 18.o (SPSS Inc., Chicago, IL, USA).

\section{RESULTS}

\section{Baseline characteristics}

The study population was comprised of 59 patients with COPD exacerbation, 61 patients with stable COPD, and 28 control subjects. The mean age of the three groups was significantly different $(73.7 \pm 8.0,70.7 \pm 5.6$, and $66.8 \pm$ 4.8 years, respectively; $p<0.001$ ). The BMI of the control group was significantly higher than patients with COPD exacerbation and stable COPD $(20.5 \pm 3.5,22.0 \pm 3.5$, and $23.8 \pm 3.2 \mathrm{~kg} / \mathrm{m}^{2}$, respectively; $p<0.001$ ). $\mathrm{FEV}_{1}$ (\% predicted) and $\mathrm{FEV}_{1} / \mathrm{FVC}$ ratio (\%) were not significantly different in patients with COPD exacerbation compared with those with stable COPD ( $\mathrm{FEV}_{1}: 52.3 \% \pm 26.0 \%$ vs. $58.5 \% \pm$ $21.6 \%$ predicted, $p=0.158 ; \mathrm{FEV}_{1} / \mathrm{FVC}: 46.8 \% \pm 13.1 \%$ vs.

Table 1. Baseline characteristics of enrolled subjects

\begin{tabular}{|c|c|c|c|c|}
\hline Characteristic & COPD exacerbation & Stable COPD & Control & $p$ value \\
\hline Subject & 59 & 61 & 28 & \\
\hline Age, yr & $73.7 \pm 8.0$ & $70.7 \pm 5.6$ & $66.8 \pm 4.8$ & $<0.001$ \\
\hline Sex, male:female & $54: 5$ & $57: 4$ & 28:0 & 0.297 \\
\hline Body mass index, $\mathrm{kg} / \mathrm{m}^{2}$ & $20.5 \pm 3.5$ & $22.0 \pm 3.5$ & $23.8 \pm 3.2$ & $<0.001$ \\
\hline Smoking status & & & & 0.106 \\
\hline Non-smoker & ० & o & o & \\
\hline Ex-smoker & $46(78.0)$ & $46(75 \cdot 4)$ & $16(57.1)$ & \\
\hline Current smoker & $13(22.0)$ & $15(24 \cdot 6)$ & $12(42.9)$ & \\
\hline Smoking history, pack-yr & $43.9 \pm 20.5$ & $38.1 \pm 13.7$ & NA & 0.074 \\
\hline $\mathrm{FEV}_{1}, \%$ predicted & $52.3 \pm 26.0$ & $58.5 \pm 21.6$ & $93.1 \pm 18.7$ & $<0.001$ \\
\hline $\mathrm{FEV}_{1} / \mathrm{FVC}, \%$ & $46.8 \pm 13.1$ & $48.1 \pm 11.1$ & $80.7 \pm 9.2$ & $<0.001$ \\
\hline GOLD stage & & & NA & 0.032 \\
\hline 1 & $8(13.6)$ & $10(16.4)$ & & \\
\hline 2 & $18(30.5)$ & $25(41.0)$ & & \\
\hline 3 & $23(39.0)$ & $25(41.0)$ & & \\
\hline 4 & $10(16.9)$ & $1(1.6)$ & & \\
\hline Frequency of exacerbation during previous year & $1.7 \pm 0.9$ & $1.8 \pm 2.6$ & NA & 0.944 \\
\hline Neutrophil to lymphocyte ratio & $12.4 \pm 10.6$ & $2.4 \pm 0.7$ & $1.4 \pm 0.5$ & $<0.001$ \\
\hline C-reactive protein, mg/L & $87.5 \pm 86.4$ & NA & $0.9 \pm 1.4$ & $<0.001$ \\
\hline
\end{tabular}

Values are presented as mean \pm SD or number (\%).

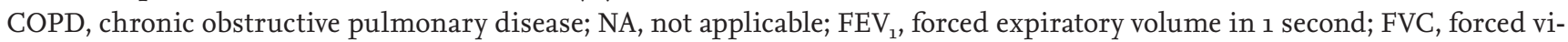
tal capacity; GOLD, Global Initiative for Chronic Obstructive Lung Disease. 
$48.1 \% \pm 11.1 \%, p=0.565)$. Frequency of COPD exacerbations during the previous year between the COPD exacerbation group and the stable COPD group was comparable. Male to female ratio and smoking status of the three groups were not significantly different (Table 1).

\section{Differences in NLR in the three groups and changes in NLR in the COPD exacerbation group}

NLR values were significantly higher in patients with COPD exacerbation compared with those with stable COPD and control subjects $(12.4 \pm 10.6,2.4 \pm 0.7$, and 1.4 \pm 0.5 , respectively; $p<0.001$ ) (Fig. 1). The differences in NLR between patients with stable COPD and controls were not significant using one-way analysis of variance and the Bonferroni post hoc test, but the differences were significant using an unpaired $t$ test $(p<0.001)$. Repetitive measurement of NLR in the convalescent period was completed in 52 of the 59 patients with COPD exacerbation. The median interval of measuring NLR between the exacerbation and the convalescent period was 18.5 days. Patients in the convalescent period had a significantly decreased NLR value compared with those in the exacerbation period ( $4.5 \pm 4.6$ vs. $11.5 \pm 8.8, p<0.001$ ) (Fig. 2 ).

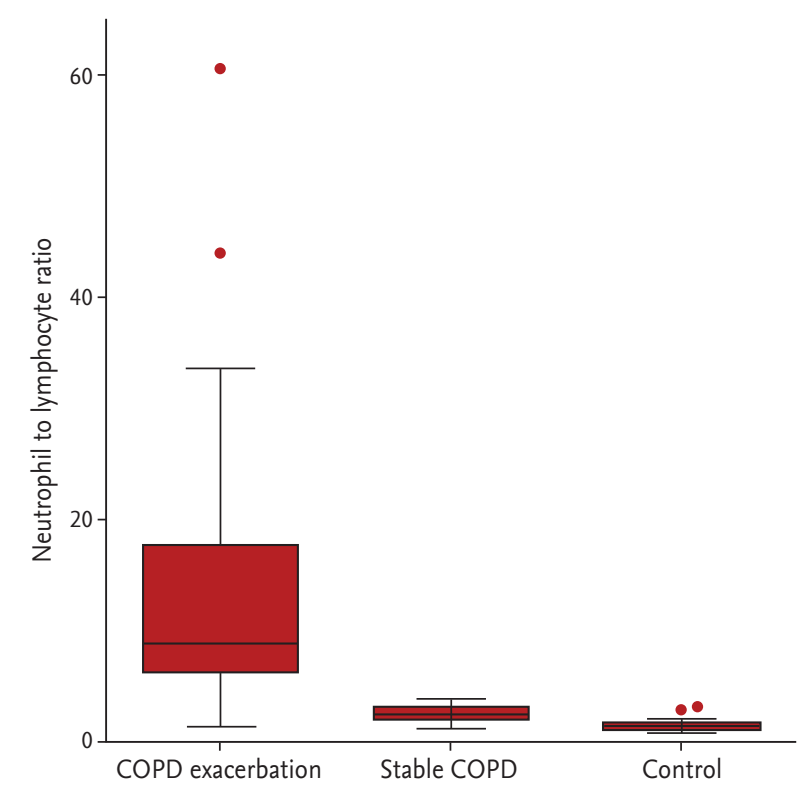

Figure 1. Neutrophil to lymphocyte ratio in patients with chronic obstructive pulmonary disease (COPD) exacerbation, stable COPD, and controls. Box plots represent median, inter-quartile range, and range with outliers plotted separately.

\section{Correlation between NLR and clinical parameters}

A total of 41 subjects, including 33 patients with stable COPD and eight patients with COPD exacerbation in the convalescent period completed the 6MWT, and thus the BODE index was calculated. Correlations of NLR with BMI and $\mathrm{FEV}_{1}$ were not significant (BMI: $r=-0.262$, $\left.p=0.098 ; \mathrm{FEV}_{1}: r=-0.285, p=0.071\right)$. However, there were significant correlations between NLR and $\mathrm{mMRC}$, and between NLR and 6MWT (mMRC: $r=0.396, p=0.01$; 6MWT: $r=-0.381, p=0.014$ ) (Fig. $3 \mathrm{~A}$ and $3 \mathrm{~B}$ ). The correlation coefficient between NLR and the BODE index was higher than the correlation coefficient between NLR and BMI, $\mathrm{FEV}_{1}$, mMRC, or 6MWT $(r=0.458, p=0.003)$ (Fig. 3C).

\section{Predictors for respiratory hospitalization}

An NLR of 2.8 was considered the optimal cut-off value with maximal sensitivity and specificity for respiratory hospitalization following ROC curve analysis. The sensitivity and specificity were $60.0 \%$ and $60.9 \%$, respectively (Fig. 4). The results of the univariate logistic regression analysis indicated that NLR $\geq 2.8$, low BMI, and low FEV were predictors of respiratory hospitalization. Low BMI (OR, $0.857 ; p=0.019)$ and low $\mathrm{FEV}_{1}(\mathrm{OR}, 0.979 ; p=0.034)$ retained significance following multivariate analysis. NLR $\geq 2.8$ was an independent predictor of respiratory

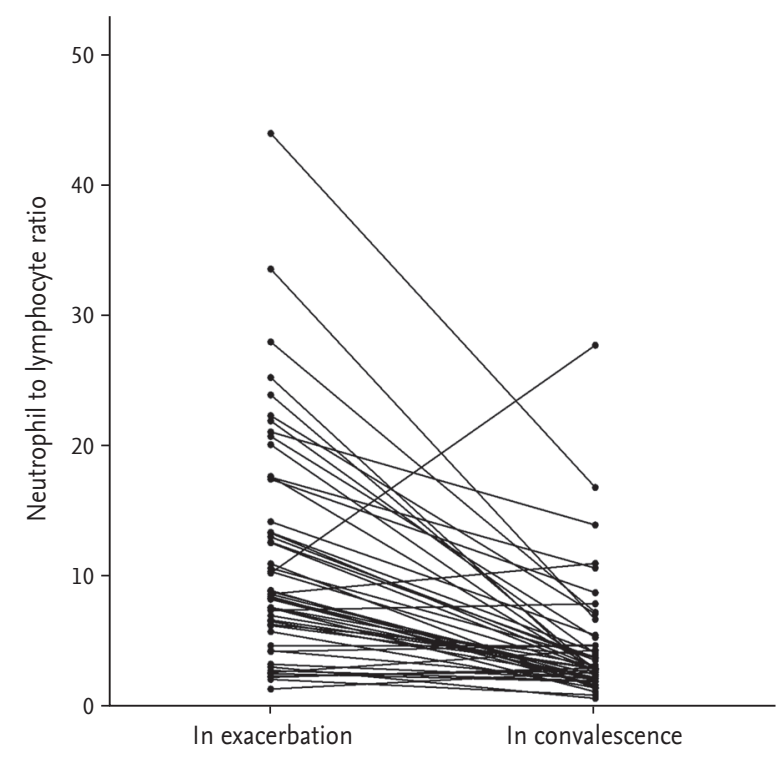

Figure 2. Change in neutrophil to lymphocyte ratio between the exacerbation and convalescence periods. 

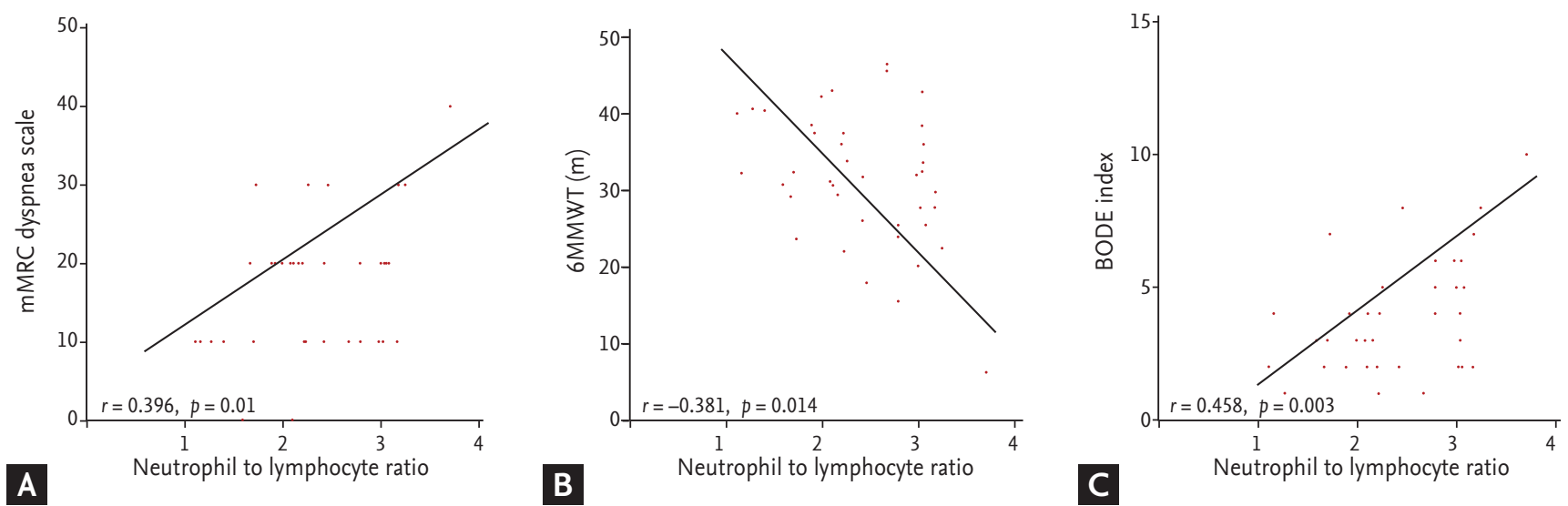

Figure 3. Correlation between neutrophil to lymphocyte ratio (NLR) and clinical parameters. (A) NLR and modified Medical Research Council scale (mMRC), (B) NLR and 6-minute walk test (6MWT), and (C) NLR and the body mass index, degree of airway obstruction, dyspnea, and exercise capacity (BODE) index.

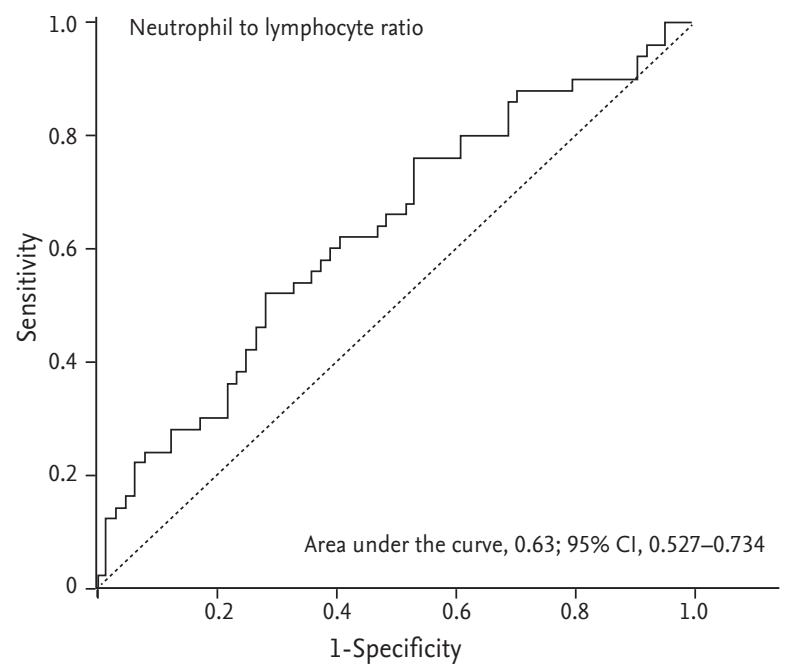

Figure 4. Receiver operator characteristics curve of neutrophil to lymphocyte ratio to predict respiratory hospitalization. CI, confidence interval.

hospitalization with borderline significance (OR, 2.083; $p=0.079$ ) (Table 2).

\section{DISCUSSION}

The current study was conducted to evaluate the usefulness of NLR as a predictive biomarker in patients with COPD. NLR was significantly higher in patients with COPD exacerbation than in controls and individuals with stable COPD. NLR values were decreased in the convalescent period of patients with COPD exacerbation. The BODE index, mMRC, and the 6MWT were significantly correlated with NLR. Low BMI and low $\mathrm{FEV}_{1}$ were predictors of respiratory hospitalization after adjusting for confounding variables. NLR $\geq 2.8$ tended to increase the odds for respiratory hospitalization.

COPD is a chronic inflammatory disease of the airways and lungs that leads to persistent airway limitation [2]. Since early 2000 , increasing evidence has indicated that COPD is associated with systemic inflammation and systemic extra-pulmonary features, or so-called comorbidities [17-19]. Agusti [20] described COPD as a multicomponent disease, and reported that systemic inflammation played a pivotal role in the development of COPD comorbidities. However, the exact mechanisms behind the development of systemic inflammation in patients with COPD remain unclear. Nonetheless, it appears that systemic inflammation in patients with COPD is associated with various comorbidities including CVD, lung cancer, cachexia, and osteoporosis [18,21-25]. Gan et al. [19] reported that various systemic inflammatory markers including C-reactive protein (CRP), fibrinogen, leukocytes, tumor necrosis factor $\alpha$, and interleukin 6 (IL-6) and IL-8 were elevated in patients with COPD. NLR is a conventional biomarker of systemic inflammation, and prognostic values of NLR have been reported in patients with lung cancer, CVD, and osteoporosis [16,26-28]. The current study showed that NLR values were higher in patients with stable COPD compared with healthy smokers, which suggested an augmented systemic inflammatory response in COPD.

An exacerbation of COPD is an important condition because it deteriorates the patient's quality of life, accel- 
Table 2. Predicting factors for respiratory hospitalization in patients with chronic obstructive pulmonary disease

\begin{tabular}{|c|c|c|c|c|c|c|}
\hline \multirow{2}{*}{ Factor } & \multicolumn{3}{|c|}{ Univariate analysis } & \multicolumn{3}{|c|}{ Multivariate analysis } \\
\hline & OR & $95 \% \mathrm{CI}$ & $p$ value & OR & $95 \%$ CI & $p$ value \\
\hline \multicolumn{7}{|l|}{ NLR } \\
\hline$<2.8$ & 1 & & & 1 & & \\
\hline$\geq 2.8$ & 2.302 & $1.081-4.902$ & 0.031 & 2.083 & $0.918-4.723$ & 0.079 \\
\hline Age, yr & 1.050 & $0.995^{-1.108}$ & 0.078 & & & \\
\hline \multicolumn{7}{|l|}{ Sex } \\
\hline Female & 1 & & & & & \\
\hline Male & 0.918 & $0.234-3.604$ & 0.902 & & & \\
\hline Body mass index, $\mathrm{kg} / \mathrm{m}^{2}$ & 0.846 & $0.749-0.956$ & 0.007 & 0.857 & $0.753-0.975$ & 0.019 \\
\hline $\mathrm{FEV}_{1}, \%$ predicted & 0.972 & $0.954-0.990$ & 0.002 & 0.979 & $0.960-0.998$ & 0.034 \\
\hline Smoking history, pack-yr & 1.008 & $0.987-1.029$ & 0.439 & & & \\
\hline BODE index & 1.315 & $0.961-1.799$ & 0.087 & & & \\
\hline
\end{tabular}

OR, odds ratio; CI, confidence interval; NLR, neutrophil to lymphocyte ratio; $\mathrm{FEV}_{1}$, forced expiratory volume in 1 second; BODE, body mass index, degree of airway obstruction, dyspnea, and exercise capacity.

erates the rate of lung function decline, and increases hospitalization frequency and mortality [2]. Because of the importance of COPD exacerbation, various plasma biomarkers have been studied. Hurst et al. [29] reported that biomarkers such as CRP, IL-6, pulmonary and activation-regulated chemokines, myeloid progenitor inhibitory factor-1, soluble intercellular adhesion molecule-1, and adiponectin were significantly elevated during exacerbation, and that CRP was the most reliable biomarker for confirming COPD exacerbation. This study likewise revealed that COPD patients with high NLR may have a higher risk of exacerbation that required hospitalization, and that NLR was significantly higher during the exacerbation period compared with the convalescence period. Considering simplicity, ease, and economical measurement in combination with the results of the study, NLR may potentially serve as a biomarker of COPD exacerbation.

The BODE index is a multidimensional index initially constructed by Celli et al. [30] to predict the risk of death among patients with COPD. In addition to the ability to predict mortality, the BODE index is a good indicator of COPD exacerbation [31]. Previous studies have demonstrated an association between the BODE index and systemic inflammatory biomarkers [32-34]. Among the four components of the BODE index, $\mathrm{mMRC}$, and 6MWT showed significant correlation with NLR, whereas no correlation was identified between NLR and BMI or $\mathrm{FEV}_{1}$. The correlation of the BODE index with NLR was both significant and more prominent than the correlation of NLR with other individual parameters in this study. Despite the fact that this study did not determine the usefulness of NLR in predicting mortality of patients with COPD, the potential role of NLR as a prognostic biomarker of COPD may exist.

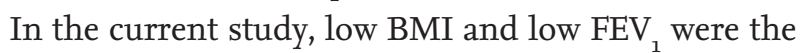
two independent risk factors for hospitalization due to COPD exacerbation. Hallin et al. [35] reported that low $\mathrm{BMI}$ and weight change were related to a higher risk of COPD exacerbation. Likewise, Pouw et al. [36] reported that low BMI and weight loss during hospitalization were risk factors for unplanned readmission. These results are in accord with the results of this study, and indicate that further efforts to improve the nutritional status of patients with COPD are needed. The degree of airway obstruction expressed by $\mathrm{FEV}_{1}$ is a validated risk factor for detrimental COPD outcomes, including exacerbation and mortality [37,38]. NLR $\geq 2.8$ was a significant predictor of respiratory hospitalization following the univariate analysis, but the significance was marginal after the multivariate analysis. To the best of our knowledge, this is the first study to evaluate the role of NLR in predicting respiratory hospitalization in patients with COPD. However, the optimal cut-off value of NLR for predicting hospitalization remains uncertain, and thus further studies are required. 
The limitations of the current study included the relatively small number of enrolled subjects, the difficulty of age-matching the subjects within the three groups, cases of mild COPD exacerbation were not verified, and the BODE index was calculated in only 41 patients. Despite these limitations, this is the first prospective observational study that evaluated the association between NLR and the BODE index, as well as the role of NLR in predicting respiratory hospitalization.

In conclusion, the potential role of NLR as a biom arker of COPD and its exacerbation is patently clear. However, future studies involving a larger number of patients are required for further confirmation.

\section{KEY MESSAGE}

1. Neutrophil to lymphocyte ratio (NLR) was higher in patients with chronic obstructive pulmonary disease (COPD) exacerbation compared with those with stable COPD and healthy controls, and decreased in convalescent period of COPD exacerbation.

2. NLR showed a significant correlation with the 6-minute walking distance, the modified Medical Research Council scale, and the BODE index.

3. NLR was an independent predictor with a borderline significance for respiratory hospitalization in patients with COPD.

\section{Conflict of interest}

No potential conflict of interest relevant to this article was reported.

\section{REFERENCES}

1. World Health Organization. The top 10 causes of death [Internet]. Geneva: World Health Organization, c2015 [cited 2015 Dec 10]. Available from: http://who.int/mediacentre/ factsheets/fs310/en/.

2. Vestbo J, Hurd SS, Agusti AG, et al. Global strategy for the diagnosis, management, and prevention of chronic obstructive pulmonary disease: GOLD executive summary.
Am J Respir Crit Care Med 2013;187:347-365.

3. Franciosi LG, Page CP, Celli BR, et al. Markers of disease severity in chronic obstructive pulmonary disease. Pulm Pharmacol Ther 2006;19:189-199.

4. Franciosi LG, Page CP, Celli BR, et al. Markers of exacerbation severity in chronic obstructive pulmonary disease. Respir Res 2006;7:74.

5. Barnes PJ, Chowdhury B, Kharitonov SA, et al. Pulmonary biomarkers in chronic obstructive pulmonary disease. Am J Respir Crit Care Med 2006;174:6-14.

6. Sinden NJ, Stockley RA. Systemic inflammation and comorbidity in COPD: a result of 'overspill' of inflammatory mediators from the lungs? Review of the evidence. Thorax 2010;65:930-936.

7. Walter RE, Wilk JB, Larson MG, et al. Systemic inflammation and COPD: the Framingham Heart Study. Chest 2008;133:19-25.

8. Vestbo J. Systemic inflammation and progression of COPD. Thorax 2007;62:469-470.

9. Williams KA, Labidi-Galy SI, Terry KL, et al. Prognostic significance and predictors of the neutrophil-to-lymphocyte ratio in ovarian cancer. Gynecol Oncol 2014;132:542550.

10. Roxburgh CS, McMillan DC. Role of systemic inflammatory response in predicting survival in patients with primary operable cancer. Future Oncol 2010;6:149-163.

11. Kacan T, Babacan NA, Seker M, et al. Could the neutrophil to lymphocyte ratio be a poor prognostic factor for non small cell lung cancers? Asian Pac J Cancer Prev 2014;15:2089-2094.

12. Walsh SR, Cook EJ, Goulder F, Justin TA, Keeling NJ. Neutrophil-lymphocyte ratio as a prognostic factor in colorectal cancer. J Surg Oncol 2005;91:181-184.

13. Azab B, Bhatt VR, Phookan J, et al. Usefulness of the neutrophil-to-lymphocyte ratio in predicting short- and long-term mortality in breast cancer patients. Ann Surg Oncol 2012;19:217-224.

14. Stotz M, Gerger A, Eisner F, et al. Increased neutrophil-lymphocyte ratio is a poor prognostic factor in patients with primary operable and inoperable pancreatic cancer. Br J Cancer 2013;109:416-421.

15. Guasti L, Dentali F, Castiglioni L, et al. Neutrophils and clinical outcomes in patients with acute coronary syndromes and/or cardiac revascularisation: a systematic review on more than 34,000 subjects. Thromb Haemost 2011;106:591-599. 
16. Bhat T, Teli S, Rijal J, et al. Neutrophil to lymphocyte ratio and cardiovascular diseases: a review. Expert Rev Cardiovasc Ther 2013;11:55-59.

17. MacNee W. Systemic inflammatory biomarkers and co-morbidities of chronic obstructive pulmonary disease. Ann Med 2013;45:291-300.

18. Agustí AG, Noguera A, Sauleda J, Sala E, Pons J, Busquets $X$. Systemic effects of chronic obstructive pulmonary disease. Eur Respir J 2003;21:347-360.

19. Gan WQ, Man SF, Senthilselvan A, Sin DD. Association between chronic obstructive pulmonary disease and systemic inflammation: a systematic review and a meta-analysis. Thorax 2004;59:574-580.

20. Agusti AG. COPD, a multicomponent disease: implications for management. Respir Med 2005;99:670-682.

21. Berger JS, Sanborn TA, Sherman W, Brown DL. Effect of chronic obstructive pulmonary disease on survival of patients with coronary heart disease having percutaneous coronary intervention. Am J Cardiol 2004;94:649-651.

22. Curkendall SM, DeLuise C, Jones JK, et al. Cardiovascular disease in patients with chronic obstructive pulmonary disease, Saskatchewan Canada cardiovascular disease in COPD patients. Ann Epidemiol 2006;16:63-70.

23. Mannino DM, Aguayo SM, Petty TL, Redd SC. Low lung function and incident lung cancer in the United States: data from the first National Health and Nutrition Examination Survey follow-up. Arch Intern Med 2003;163:14751480.

24. Schols AM. Pulmonary cachexia. Int J Cardiol 2002;85:101110.

25. Biskobing DM. COPD and osteoporosis. Chest 2002;121:609620.

26. Yao Y, Yuan D, Liu H, Gu X, Song Y. Pretreatment neutrophil to lymphocyte ratio is associated with response to therapy and prognosis of advanced non-small cell lung cancer patients treated with first-line platinum-based chemotherapy. Cancer Immunol Immunother 2013;62:471479.

27. Yilmaz H, Uyfun M, Yilmaz TS, et al. Neutrophil-lymphocyte ratio may be superior to C-reactive protein for predicting the occurrence of postmenopausal osteoporosis.
Endocr Regul 2014;48:25-33.

28. Kang MH, Go SI, Song HN, et al. The prognostic impact of the neutrophil-to-lymphocyte ratio in patients with small-cell lung cancer. Br J Cancer 2014;111:452-460.

29. Hurst JR, Donaldson GC, Perera WR, et al. Use of plasma biomarkers at exacerbation of chronic obstructive pulmonary disease. Am J Respir Crit Care Med 2006;174:867874.

30. Celli BR, Cote CG, Marin JM, et al. The body-mass index, airflow obstruction, dyspnea, and exercise capacity index in chronic obstructive pulmonary disease. N Engl J Med 2004;350:1005-1012.

31. Marin JM, Carrizo SJ, Casanova C, et al. Prediction of risk of COPD exacerbations by the BODE index. Respir Med 2009;103:373-378.

32. Pinto-Plata V, Toso J, Lee K, et al. Profiling serum biomarkers in patients with COPD: associations with clinical parameters. Thorax 2007;62:595-601.

33. Liu SF, Chin CH, Wang CC, Lin MC. Correlation between serum biomarkers and BODE index in patients with stable COPD. Respirology 2009;14:999-1004.

34. Gaki E, Kontogianni K, Papaioannou AI, et al. Associations between BODE index and systemic inflammatory biomarkers in COPD. COPD 2011;8:408-413.

35. Hallin R, Koivisto-Hursti UK, Lindberg E, Janson C. Nutritional status, dietary energy intake and the risk of exacerbations in patients with chronic obstructive pulmonary disease (COPD). Respir Med 2006;100:561-567.

36. Pouw EM, Ten Velde GP, Croonen BH, Kester AD, Schols AM, Wouters EF. Early non-elective readmission for chronic obstructive pulmonary disease is associated with weight loss. Clin Nutr 2000;19:95-99.

37. Bahadori K, FitzGerald JM. Risk factors of hospitalization and readmission of patients with COPD exacerbation: systematic review. Int J Chron Obstruct Pulmon Dis 2007;2:241-251.

38. inganayagam A, Schembri S, Chalmers JD. Predictors of mortality in hospitalized adults with acute exacerbation of chronic obstructive pulmonary disease. Ann Am Thorac Soc 2013;10:81-89. 